\title{
Disruption of bacterial cell-to-cell communication by marine organisms and its relevance to aquaculture.
}

\begin{abstract}
Bacterial disease is one of the most critical problems in commercial aquaculture. Although various methods and treatments have been developed to curb the problem, yet they still have significant drawbacks. A novel and environmental-friendly approach in solving this problem is through the disruption of bacterial communication or quorum sensing (QS). In this communication scheme, bacteria regulate their own gene expression by producing, releasing, and sensing chemical signals from the environment. There seems to be a link between QS and diseases through the regulation of certain phenotypes and the induction of virulence factors responsible for pathogen-host association. Several findings have reported that numerous aquatic organisms such as micro-algae, macro-algae, invertebrates, or even other bacteria have the potential to disrupt QS. The mechanism of action varies from degradation of signals through enzymatic or chemical inactivation to antagonistic as well as agonistic activities. This review focuses on the existing marine organisms that are able to interfere with QS with potential application for aquaculture as bacterial control.
\end{abstract}

Keyword: Quorum sensing inhibitors; Quorum sensing interference; Inhibition; Quenching; Marine organisms; Aquaculture. 